\title{
Ultrahigh angular selectivity of disorder-engineered metasurfaces
}

\author{
Mohammad Haghtalab ${ }^{1,2^{*}}$, Michele Tamagnone ${ }^{1}$, Alexander Y. Zhu ${ }^{1}$, Safieddin \\ Safavi-Naeini ${ }^{2}$, and Federico Capasso ${ }^{1}$ \\ ${ }^{1}$ John A. Paulson School of Engineering and Applied Sciences, Harvard University, Cambridge, \\ MA 02138 USA \\ ${ }^{2}$ Department of Electrical and Computer Engineering, University of Waterloo, Waterloo, ON \\ N2L 3 G1 Canada \\ *Email: mhaghtalab@g.harvard.edu; mhaghtal@uwaterloo.ca
}

S1: Disorder-induced Localization

S2: The Guided Waves Localization

S3: Localization Assisted Beamforming

S4: Design sensitivity with respect to the locations and geometrical sizes of scattering elements S5: Near Optimal Freeform Inverse Design

Figures:

Fig. S1: Singular value decomposition (SVD) analysis: The probability distribution function (PDF) of the localization length and the singular value distribution for disordered and periodic metasurfaces.

Fig. S2: The localization of guided wave due to radiation and disorder in disordered and periodic metasurfaces.

Fig. S3: Localization assisted beamforming : study on designed metasurface of Figure 3 (a) in the paper.

Fig. S4: The evolution of electric fields inside the elements: study on the designed metasurface of Figure 3 (a) in the paper.

Fig. S5: The probability distribution functions of the drop in efficiency due to displacement and geometrical errors of elements: study on the metasurface designs of the Figure 3 (a), and (b) of the paper.

Fig. S6: Ten different realizations for the design target of Figure 3(a) of the paper. 


\section{S1: Disorder-induced Localization}

The induced fields inside the scattering elements can be described in terms of the incident fields through the matrix expression $[\alpha]=[Z]^{-1}\left[\mathbf{E}_{\mathbf{i n c}}\right]$, where $\left[\mathbf{E}_{\mathbf{i n c}}\right]$ and $[\alpha]$ give the incident and total electric fields, respectively, inside the scattering elements, and $[Z]$ describes the inter- and intraelement couplings. As explained in the Design Section of paper, we employ the method of moments (MoM) to calculate the matrix elements. The analysis of the singular value decomposition (SVD) of $[Z]^{-1}=[U][\Sigma][V]^{T}$ provides insights about the effect of randomness on the distribution of induced fields inside the scattering elements in terms of the eigenstates of the structure. Assuming that we have a structure consisting of $N$ sub-wavelength elements and $[Z]$ is an $N \times N$ matrix, then $[\mathrm{V}]=\left[\mathrm{V}_{1}\left|\mathrm{~V}_{2}\right| \ldots \mid \mathrm{V}_{\mathrm{N}}\right]$ and $[\mathrm{U}]=\left[\mathrm{U}_{1}\left|\mathrm{U}_{2}\right| \ldots \mid \mathrm{U}_{\mathrm{N}}\right]$ are unitary square matrices where the column vectors of $[\mathrm{V}]([\mathrm{U}])$ are the eigenvectors of $\left([Z]^{-1}\right)^{T} \times[Z]^{-1}\left([Z]^{-1}\right.$ $\left.\times\left([Z]^{-1}\right)^{T}\right)$. The singular values are the diagonal elements of $[\Sigma]$ which is an $N \times N$ nonnegative real diagonal matrix with diagonal elements (singular values of $[Z]^{-1}$ ) denoted by $\sigma_{n}$ $=\sqrt{\tau_{n}}, \sigma_{1}>\sigma_{2}>\ldots>\sigma_{N}$, where $\tau_{n} \mathrm{~s}$ are the eigenvalues of $\left([Z]^{-1}\right)^{T} \times[Z]^{-1}$. From a mathematical perspective, the multiplication of incident fields, denoted by $\left[\mathbf{E}_{\text {inc }}\right]$, by $[Z]^{-1}$, first maps the input fields to the eigenvectors $\mathrm{V}_{\mathrm{n}} \mathrm{s}$, then scales the mapping coefficients by the singular values of $[Z]^{-1}$, and finally, constructs the total electric fields induced inside the scattering elements in terms of the eigenstates of the structure $\left(U_{n} s\right)$. The effective number of non-zero elements of the eigenvectors $\mathrm{V}_{\mathrm{n}}\left(\mathrm{U}_{\mathrm{n}}\right)$, defined as $\exp (H)$, where $H\left(v_{1}, \ldots, v_{N}\right)=-$ $\sum_{i=1}^{N}\left|v_{i}\right|^{2} \ln \left|v_{i}\right|^{2}, \sum_{i=1}^{N}\left|v_{i}\right|^{2}=1$, is an indication of the localization length of vectors $\mathrm{V}_{\mathrm{n}} \mathrm{s}\left(\mathrm{U}_{\mathrm{n}} \mathrm{s}\right)$, which are the eigenstates of the structure used to describe the total fields inside the elements [1].

To study the effect of disorder on the localization length of the eigenvectors, represented by $V_{n} s$ $\left(\mathrm{U}_{\mathrm{n}} \mathrm{s}\right)$, in disordered metasurfaces, we generate a large number of randomly configured metasurfaces where each realization consists of 413 scattering elements with $a=50 \mathrm{~nm}$ and $b=$ $50 \mathrm{~nm}$ distributed randomly over the range $-62 \mu \mathrm{m}<x<+62 \mu \mathrm{m}$ and located on top of a dielectric slab waveguide with a thickness of $t_{1}=65 \mathrm{~nm}$ [see Figure 1 in the paper]. The operating wavelength $(650 \mathrm{~nm})$ and materials characteristics are similar to those discussed in the Results Section of the paper. The probability distribution function (PDF) of the localization length, which is proportional to the number of non-zero elements of eigenvectors, can be obtained based on the SVD analysis explained above. The comparison with a periodic configuration with the same number of elements shows a significant reduction in the localization length of eigenvectors due to the introduction of randomness to the location of scattering elements (Fig. S1 (a)). In addition, the average of singular values taken over the large number of realizations, depicted in Figure S1 (b), suggests the enhancement of localized fields inside the scattering elements. These two distinct features of the disordered metasurfaces composed of strongly coupled elements; namely the reduced localization length of the eigenstates as well as the enhancement of localized fields, can be exploited to realize the desired field distributions both in near- and far-field regions. 


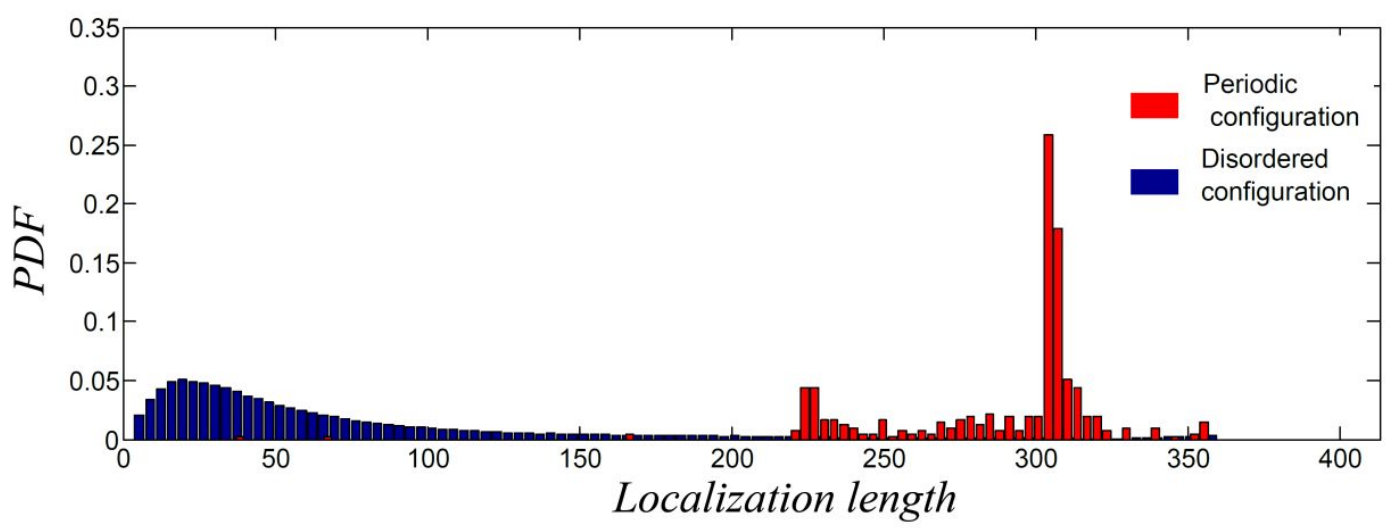

(a)

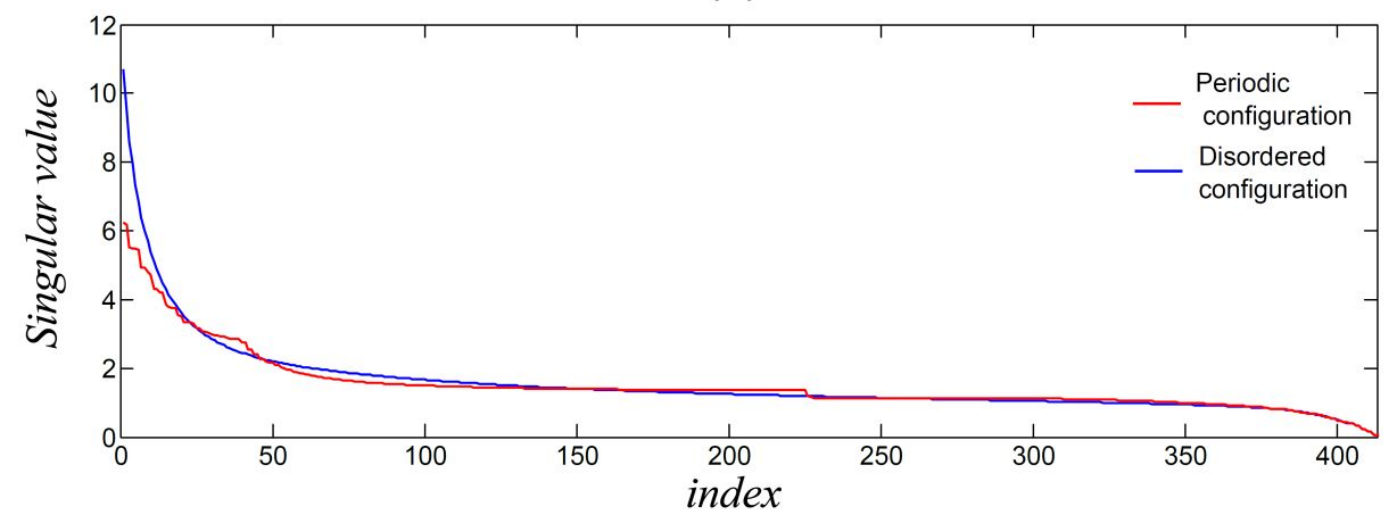

(b)

Figure S1. (a) The blue bars present the probability distribution function (PDF) of the localization length of eigenvectors of the structure generated based on SVD analysis of large number of metasurfaces, each realization consists of 413 randomly configured elements. The red bars present the distribution of eigenvectors localization length for a periodic configuration with same number of elements (413). Disorder leads to significant reduction in the localization length. (b) The blue curve presents the average of the singular values taken over large number of disordered metasurface realizations. The red curve shows the singular values of the periodic configuration.

\section{S2: The Guided Waves Localization}

In this Section, we investigate the effects of disorder and radiation loss on the propagation of guided waves through the metasurfaces. To this end, we consider geometries similar to those we studied in the Section S1. The scattering elements interact with an incident guided wave excited or launched by an illuminated element located at a far distance from the rest of the structural elements, i.e. at $x_{\text {launcher }} \ll-62 \mu \mathrm{m}$. The total field inside the launcher element is normalized to have a unity amplitude. In other words, the scattering elements are illuminated by a guided wave launched by a unity amplitude launcher located at a specific far distance from the elements. In the absence of the scattering elements, the guided wave propagation length extends to infinity (assuming negligible material loss). However, upon interaction with scattering elements, the launched guided wave undergoes multiple scattering as well as leakage losses due to the coupling to the radiating modes of free space outside the dielectric slab. The random interference 
of multiple scattered guided waves due to their interaction with randomly configured elements further enhances the localization halting the transmission of the incident guided wave through the disordered metasurfaces.

To explore the guided waves localization, we conduct study for a periodic configuration and also for a large number of randomly configured metasurfaces. The scattering elements of each configuration are illuminated by a guided wave launcher with unity amplitude located at an arbitrarily large distance from the elements at $x_{\text {launcher }}=-2.6 \mathrm{~mm}$. Simulations are carried out for all configurations and the average values of the absolute induced fields (total fields) inside the scattering elements and the total fields over a plane located at a close distance $(25 \mathrm{~nm})$ from the elements on top are calculated (Fig. S2).

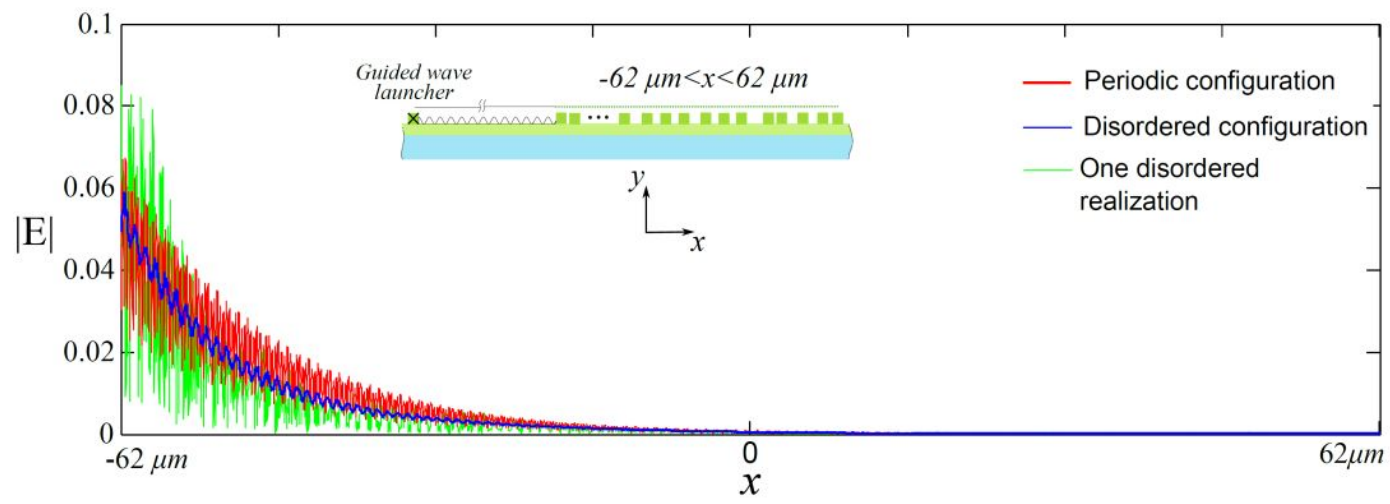

(a)

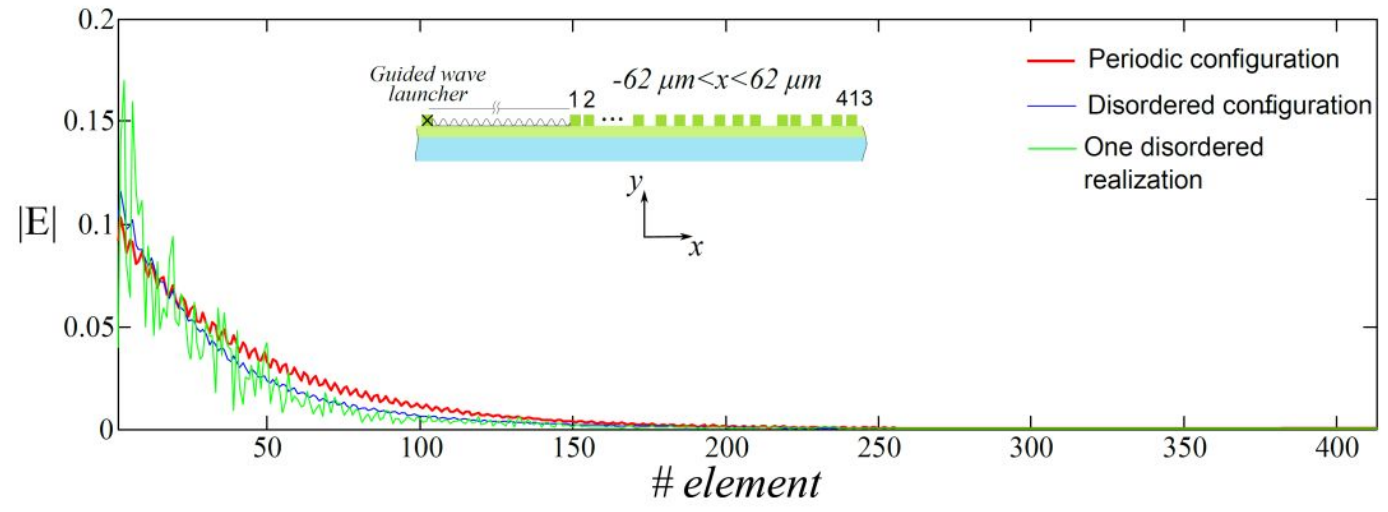

(b)

Figure S2. The absolute value of the electric field distribution over a plane at close distance, $25 \mathrm{~nm}$, above the structure, in (a), and inside the scattering elements, in (b), due to the incident guided wave on the metasurfaces. The incident guided wave is generated by a unity amplitude launcher at large distance from the elements $\left(x_{\text {launcher }}=-2.6 \mathrm{~mm}\right)$. The red curves present the results for a periodic configuration consisting of 413 elements over $-62 \mu m<x<+62 \mu m$. The radiation loss leads to localization in periodic configurations. The blue curves are the average values taken over large number of disordered metasurfaces. The green curves show the results for one particular realization. As shown, disorder leads to more localized distribution for the excited guided waves.

As it is shown, the incident guided wave will be attenuated as it propagates inside the mid-layer of metasurface while interacting with the scattering elements on top. While the loss associated 
with the radiation is the main factor causing the localization in a periodic configuration, the disorder provides more favorable conditions for the localization of guided waves in randomly configured structures.

\section{S3: Localization Assisted Beamforming}

In the paper, we demonstrated the realization of different functions through ultrahigh angular selective disordered metasurfaces. More specifically, we focused on beamforming application, where the metasurfaces are designed for directional transmission of the incident fields, illuminating the structures at specific arbitrary angle(s), towards the preset arbitrary angular direction(s). The principle of operation, here, is similar to those of leaky wave antennas or the so called holographic leaky wave metasurfaces, where the structures are fed at proper location(s) for exciting the guided waves which then are coupled to the free space desired radiating mode(s). To perform the beamforming in this type of antennas, the common approach is the frequency scanning through which the beam direction will be swept within a certain angular range. In other type of antennas, the beamforming is typically performed by applying phase shift between the signals feeding the antenna elements. As the implementation of phase shifters is challenging in certain applications especially those operating at high frequency ranges, various beam switching techniques have been proposed. The main idea has been to engineer and exploit the resonant modes of intermediate structures, such as cavities, which feed the antennas or support leaky wave modes. Using several excitation ports associated with different mode(s) of cavity provides the ability of selective choice between the desired beams in the far-field. Examples include Rotman lens antennas [2], and dual-functional holographic metasurfaces [3], to name a few. In this paper, we proposed metasurfaces featuring engineered localized modes. As we show in the following, illuminating the deigned structures with preset incident fields leads to the excitation of certain groups of modes supported by the structure. The collective effect is the formation of "virtual excitation ports" within the structure resulting in the desired beamforming operation of the metasurfaces.

In this Section, we investigate how the localization of guided waves can be harnessed to engineer the field distribution in near- and far- field regions. The metasurfaces we study in this work can be seen as transformers converting the preset incident fields into the engineered localized modes of the system composed of strongly coupled scattering elements. The localized modes are coupled back into the free space to generate the desired radiation from the metasurface. Beside the external incident fields, the scattering elements are the secondary (virtual) radiating sources, which are responsible for the generation of scattering fields from the illuminated structures. As mentioned previously, due to the coupling of elements radiations to the guided modes of the dielectric slab, the scattering elements are also considered the main launchers of guided waves within the structure. The strength of the radiated field from each element is proportional to the induced field inside it. Due to disorder and the associated localization effects, the total fields inside the elements can be described in terms of localized eigenstates (as explained in S1). In other words, as opposed to the periodic configuration with close to uniform distribution for the 
induced fields inside the elements, now, due to disorder, some elements radiate much stronger than others; we refer to these elements as key elements. The radiation from the illuminated structure can be reduced to those from the key elements which have stronger radiation than other elements. The key elements, which are the secondary (virtual) radiating sources, excite the guided waves. The rest of the elements, acting more like parasitic elements, are coupled to these virtual sources to shape the radiation in the free space outside of the metasurface. The locations of the key elements with respect to the other (parasitic) elements and the induced fields inside them are the two crucial factors in determining the final radiation from the metasurface. It is important to mention that in the multifunction metasurfaces, depending on the incident fields, the elements will serve as both key elements and parasitic elements resulting in desired radiations under different illumination conditions.

To demonstrate the explained concept, we consider structures with geometrical parameters similar to those studied in previous Sections (S1 and S2) consisting of elements with $a=50 \mathrm{~nm}$ and $b=50 \mathrm{~nm}$ distributed over the range $-62 \mu \mathrm{m}<x<+62 \mu \mathrm{m}$ and located on top of a dielectric slab waveguide with a thickness of $t_{1}=65 \mathrm{~nm}$. In the first case study, we consider metasurfaces with randomly configured elements. Illuminating the structures with normally incident plane wave, the induced (total) field inside each element is calculated. In the next step, the radiated fields from each element is calculated and the field distribution above the metasurface ( $25 \mathrm{~nm}$ above the elements) due to each single element is plotted. Figure S3 (a) shows the results for one random realization. The blue curve, on the left side, is the total induced field inside each element. There is a large random fluctuation in the field distribution. The "key elements", with strong induced field inside, are distributed randomly over the entire extent of the metasurface. As a result, the collective effect of the radiation from all the elements reduces the total radiated field in all angular directions and results in close to uniformly distributed noise-like scattered fields. Also, as can be seen, the combined effect of disorder and radiation loss prevents the large extent of the excited guided waves by each scattering element. In the second case study, we consider the design of Figure 3 (a) of the paper. Figure S3 (b) illustrates the results of the designed metasurface. The field distribution reveals the enhancement of localization in certain elements (the "key elements") due to the engineered disorder and guided wave resonances effects. As expected and is evident from the plot, the excited guided waves from the high intensity elements expand over larger length while interacting with other elements. As such, they play the critical role of transforming the incident fields into the intermediate near-fields which lead to the desired far-field radiations.

Figure S4 demonstrates the evolution of the induced electric fields inside each scattering element as the structure is modified during the design process. As can be seen, adding new scattering elements during the design process, or in other words, increasing the level of "near-optimized" disorder through the aforementioned algorithm in the paper modifies the induced electric fields inside the existing elements and improves the localization of fields inside the "key elements". Adding elements continues until no additional element can further enhance the favorable 
localizations leading to desired far-field distribution. The blue curve, in the bottom of the Figure S4, shows the absolute value of the induced fields inside the elements at the end of design process.

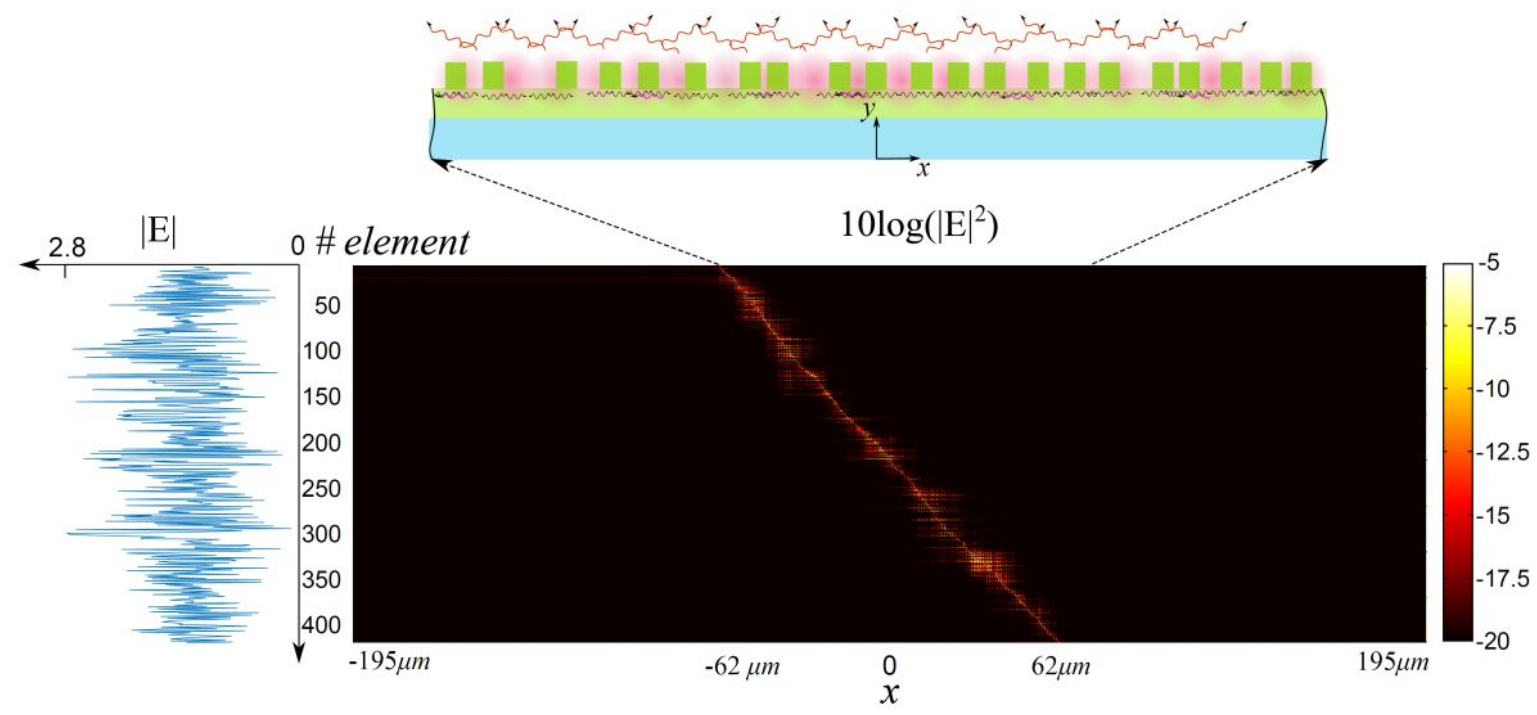

(a)

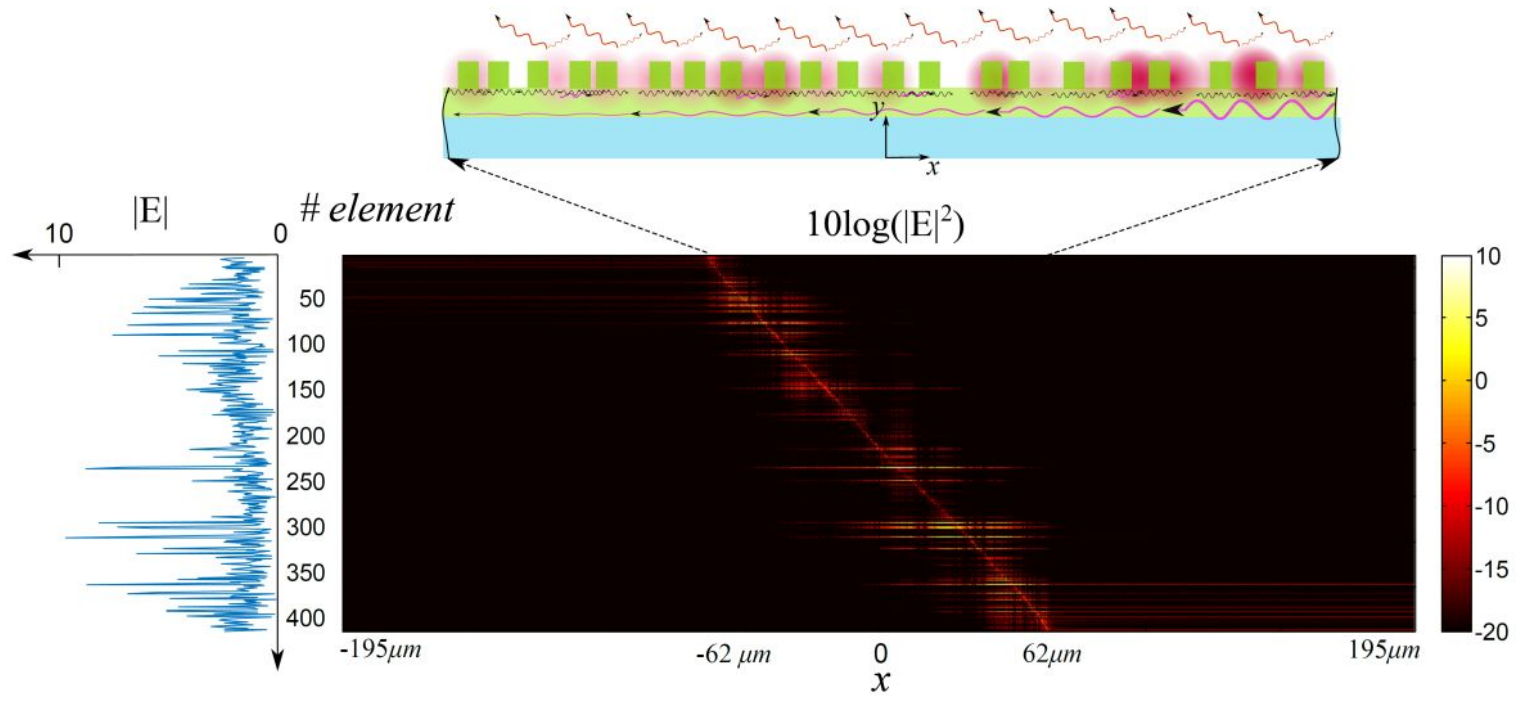

(b)

Figure S3. The left curves show the total electric field distribution inside the elements of metasurfaces, due to normally incident plane wave with unity amplitude. The right plots are each element's contribution to the electric field on top of the metasurfaces, at a plane $25 \mathrm{~nm}$ above the elements. Each element acts like a secondary source radiating in the presence of other elements. (a) The results for a metasurface with randomly configured elements. The random contributions of elements result in uniformly distributed noise-like radiation. (b) The results for the designed metasurface of Figure 3 (a) in the paper. The incident field is mostly localized in the elements located at the right half part of the metasurface launching guided waves interacting with the elements on the left part and coupled to the desired far-field beam. 


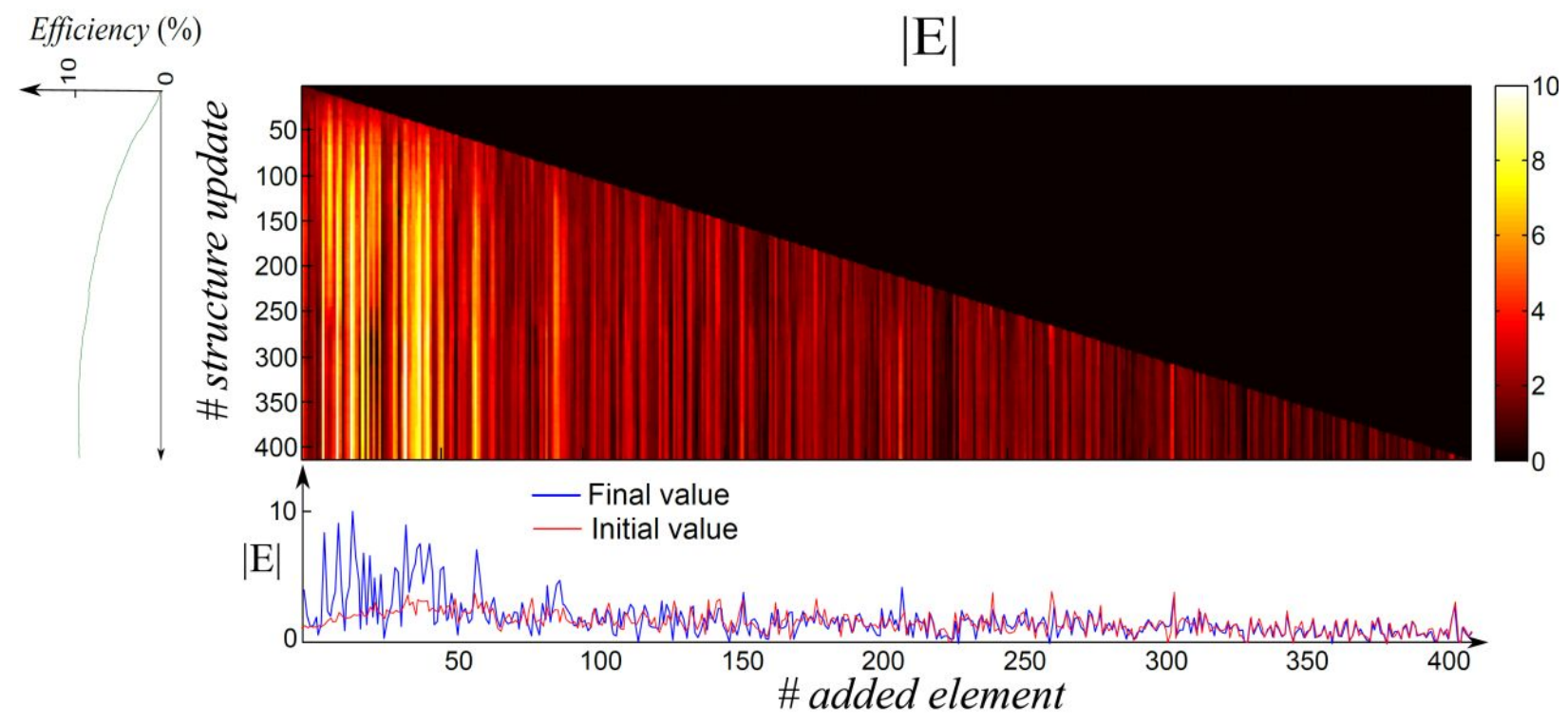

Figure S4. The evolution of induced fields inside the elements due to a normally incident plane wave with unity amplitude for the designed metasurface of Figure 3 (a) in the paper, as the elements are added to the structure. As the level of disorder increases, the enhanced localization of fields leads to higher efficiency and, equivalently, a desired far-field radiation. The horizontal axis shows the elements numbers in the sequence they are added to the structure. The structure's configuration is updated by adding each element. The vertical axis shows the number of update. In the $\mathrm{n}^{\text {th }}$ update of the structure, the $\mathrm{n}^{\text {th }}$ element is added when, due to interactions with the rest of the previously added elements, an initial electric field is induced inside it (the red curve in the bottom). The next updates to the structure change the initial induced fields. The final field distribution is shown by the blue curve.

S4: Design sensitivity with respect to the locations and geometrical sizes of scattering elements

It is of practical importance to study the design tolerability with respect to the expected fabrication errors associated with the locations and geometrical sizes of elements. For this purpose, we study the designed metasurfaces of Figure 3(a) and 3(b) of the paper consisting of 413 and 119 scattering elements with cross sectional sizes of $a=b=50 \mathrm{~nm}$, and $a=100 \mathrm{~nm}, b=300$ $\mathrm{nm}$, respectively. Performing Monte Carlo simulations, we arrive at estimated probability distribution functions (PDFs) based on different defined scenarios.

In the first scenario, we assume the errors only apply to the locations of elements. Errors up to 25 $\mathrm{nm}$, in the locations of wires, randomly drawn from a uniform distribution function from $-25 \mathrm{~nm}$ to $25 \mathrm{~nm}([-25 \mathrm{~nm} 25 \mathrm{~nm}])$ are generated and the efficiency for each realization is calculated. The results, expressed in terms of the percentage of drop in efficiency, are fit to Gaussian probability distribution functions with the same average and standard deviation of the calculated data. As shown by the purple graphs in Figure S5 (a) and (b), an average drop of 78\% and 5.5\% in efficiency is observed for the two different designs, where the first and second design are made up of lines with $a=b=50 \mathrm{~nm}$ and $a=100 \mathrm{~nm}, b=300 \mathrm{~nm}$, respectively. The corresponding standard deviation is approximately $2.8 \%$ and $4.7 \%$, respectively. The relatively large drop in 
efficiency, in the first design, is attributed to the maximum displacements with respect to the elements cross sectional sizes, i.e. $50 \%$ of $a=b=50 \mathrm{~nm}$. In the second scenario, the locations of elements are not changed and the random errors, with same probability distribution as the first scenario, only apply to the width of lines. Assuming the two designs, for each case, Monte Carlo simulations are performed. The green curves of Figure S5 present the fitted Gaussian distribution functions to each case. Errors up to $25 \mathrm{~nm}$ applied to the width of lines result in an average drop in efficiency of $87.8 \%$, and $26.1 \%$, for the two designs, respectively. The corresponding standard deviations are approximately $2 \%$, and $6.1 \%$, respectively. The results indicate that such designs are more tolerant of uncontrollable factors in fabrications leading to errors in the locations of scattering elements, but they bear higher sensitivity with respect to the errors in the geometrical sizes of them. In addition, structures consisting of larger elements are more tolerant of errors with same probability distribution characteristics.

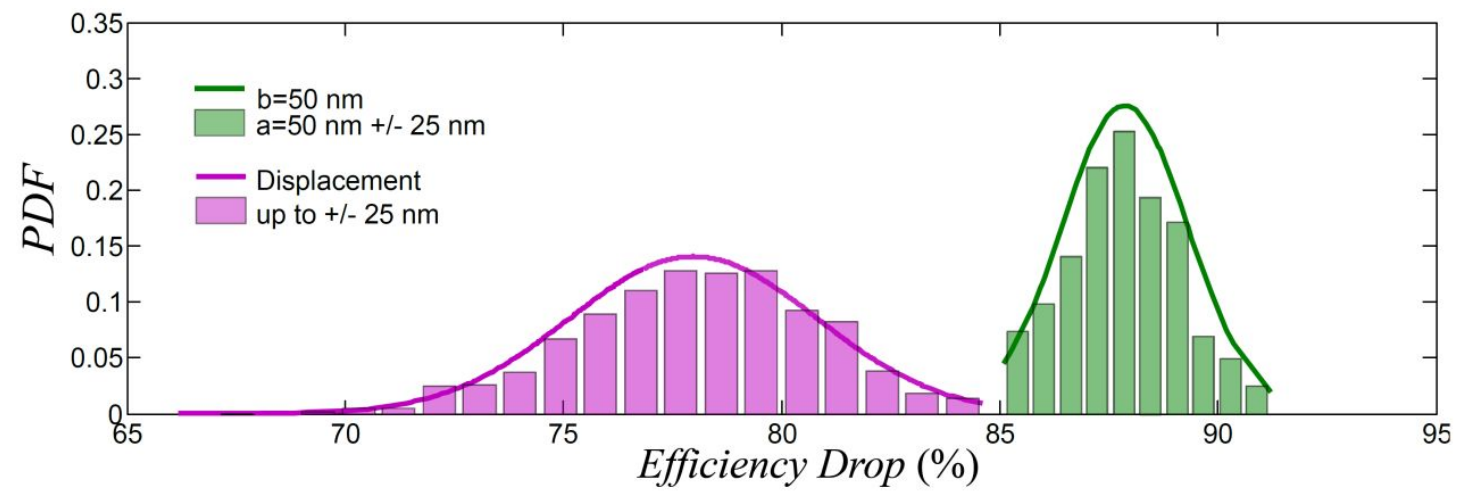

(a)

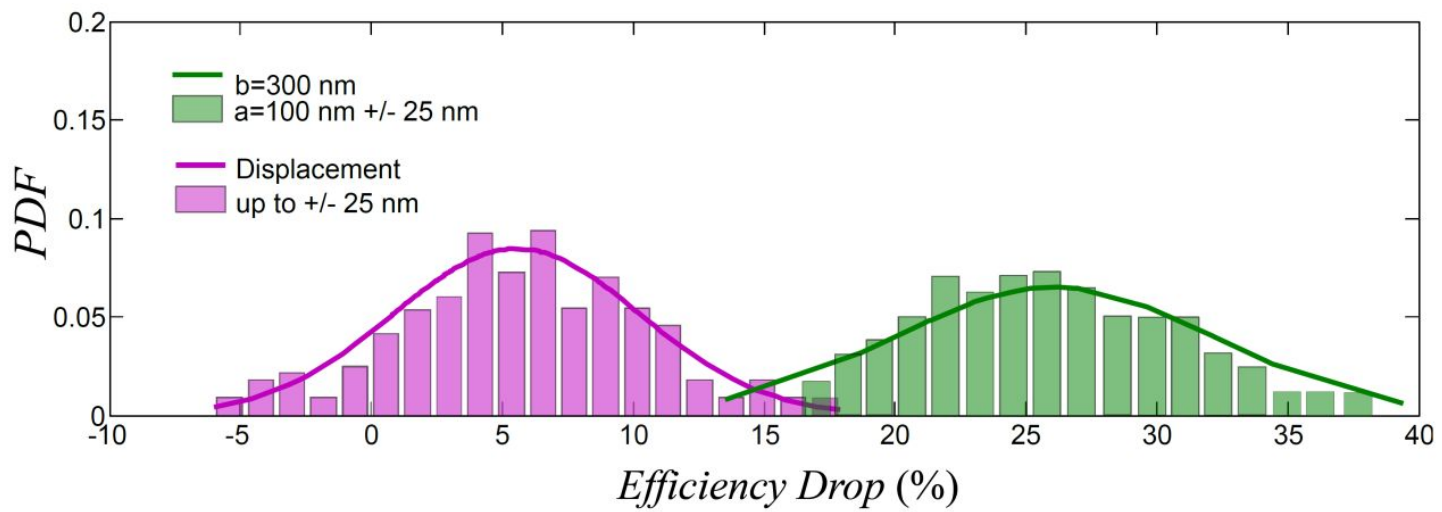

(b)

Figure S5. The fitted Gaussian probability distribution functions for the efficiency drop (\%) due to the errors in the locations (the purple graphs) and the geometrical sizes (the green graphs) of the scattering elements in the metasurface designs of Figure 3 (a) and (b) of the paper. The purple graphs are for the displacement errors up to $25 \mathrm{~nm}$, while there is no geometrical error. The green graphs are associated with the errors in the line widths up to $25 \mathrm{~nm}$, while the line locations are kept unchanged (nominal values). (a) The results for the first design consisting of elements with nominal values of $a=b=50$ $\mathrm{nm}$. (b) The results for the second design consisting of elements with nominal values of $\mathrm{a}=100 \mathrm{~nm}$ and $\mathrm{b}=300 \mathrm{~nm}$. 


\section{S5: Near Optimal Freeform Inverse Design}

In this paper, the inverse design process is performed through our developed algorithm outlined in [4]. In summary, the algorithm, which we refer to as near optimal freeform inverse design (NOFID), is based upon statistical modifications to the design by adding one sub-wavelength scattering element at a time while taking advantage of the infinitely large degrees of freedom available in the design space. Evaluating the updated cost function(s) and comparing the new value(s) with adaptively controlled threshold(s), final locations are chosen from a random pool of candidate locations for adding new elements. This process is continued until no additional element can further enhance the defined cost functions. The implemented form of the algorithm, in this paper, assumes that the scattering elements have fixed and identical geometrical parameters and only the locations of elements are unknown. Although, in a more general sense, there is no limitation in terms of considering elements with various geometrical sizes such as width or height in the design process.

It is important to note that there are several local minima (maxima) or non-unique solutions for the defined cost function in such problems which can represent different designs with acceptable figure of merit, defined through the cost function (s).Although the proposed algorithm seeks for global solutions, through taking advantage of the available large degrees of freedom, the evolution of metasurface within each design step strongly depends on its initial configuration. As such, there are infinitely large numbers of final configurations that the algorithm might converge to, which is mainly due to the randomized nature of selected configurations during the design process. A similar behavior is observed and studied for topology optimized metasurfaces [5].

Figure S6 (a) shows ten different realizations for the design target of Figure 3(a) of the paper. All the designs localize the far-field radiation around the angular direction of $-0.122 \pi$ for the normally incident plane waves. The efficiencies of different configurations are ranged from approximately $9 \%$ to $11 \%$. Although the configurations seem to be uncorrelated in the spatial domain, the Fourier transforms reveal high spectral correlations between different realizations. Figures S6 (b) and (c) show the Fourier transforms of the configurations defined as $\sum_{n=1}^{N} \exp \left(-j k x^{m}[n]\right)$, where $x^{m}[n]$ is the center of the $\mathrm{n}^{\text {th }}$ scattering element in the $\mathrm{m}^{\text {th }}$ realization. As expected, the elements of various configurations generate the required spatial frequencies (diffraction orders) including the desired angular directions and the guided waves components. As mentioned previously, the engineered disorder is the key factor modifying the induced fields inside the elements so to suppress the unwanted existing diffraction orders seen in Figure S6 (b) and (c). 


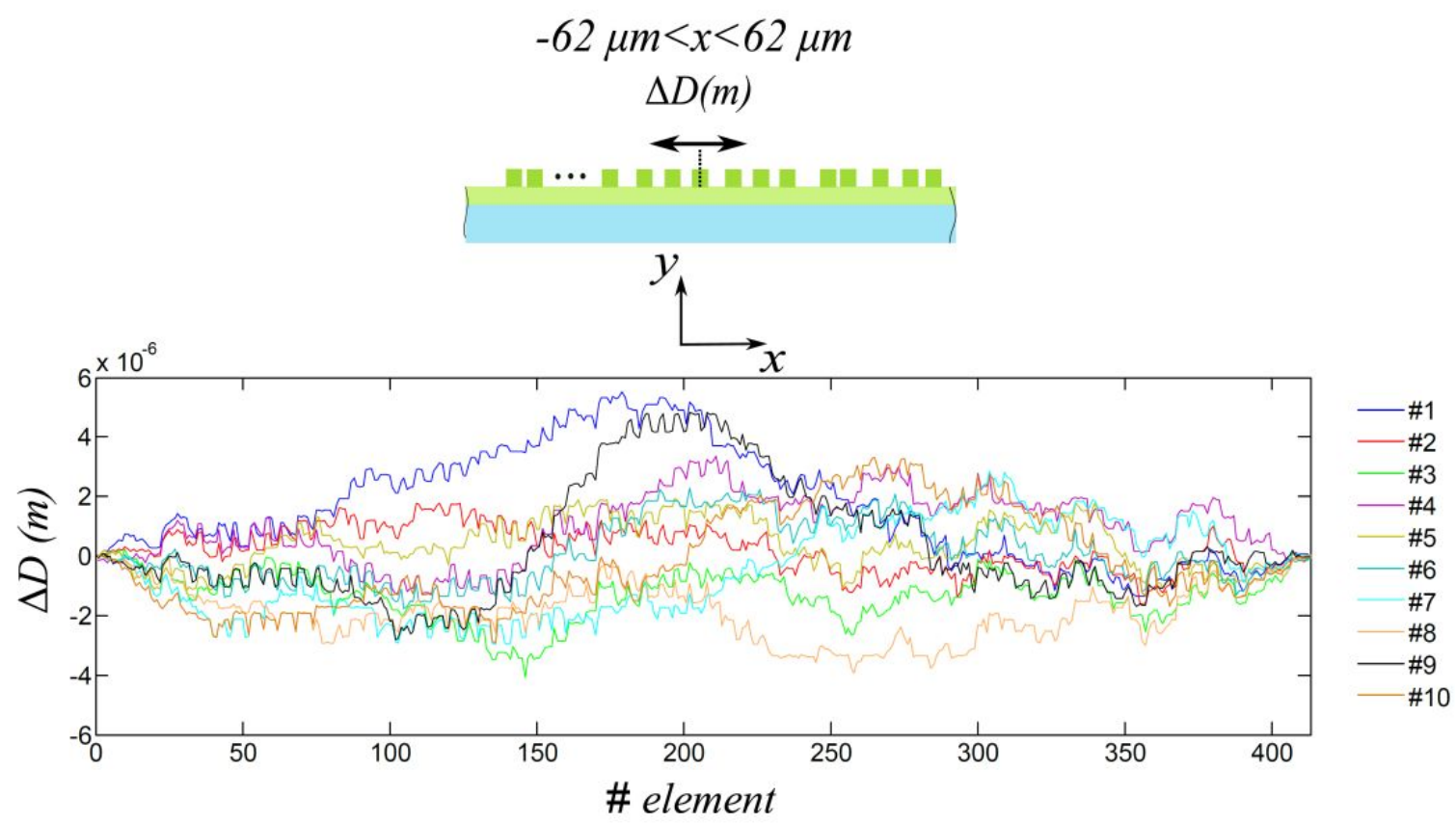

(a)

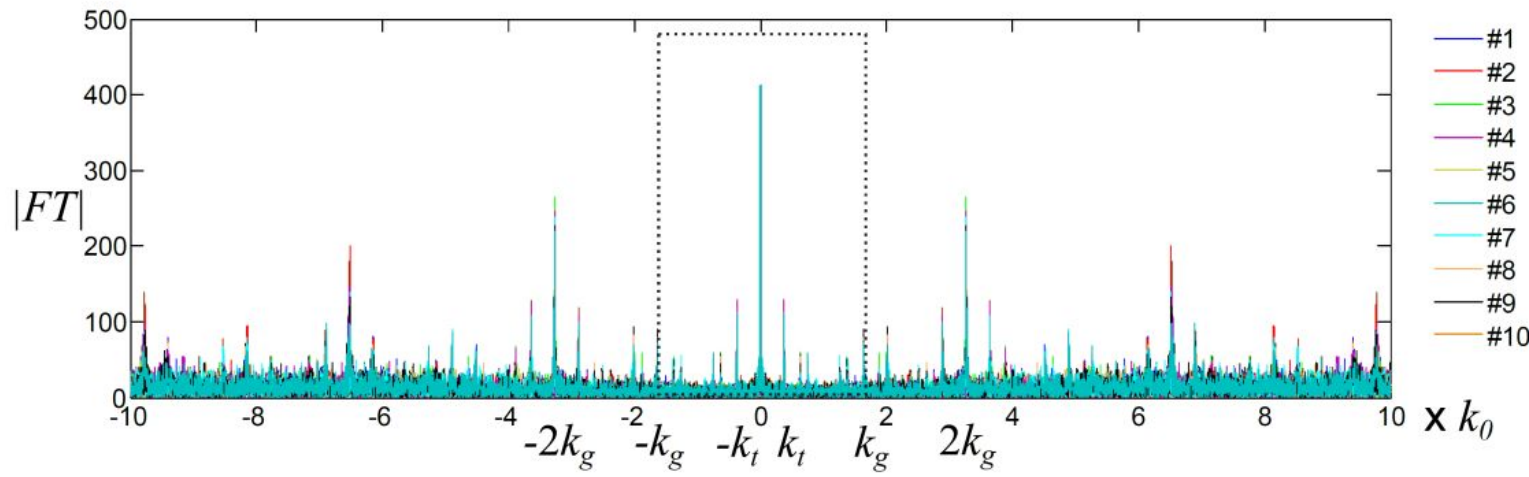

(b)

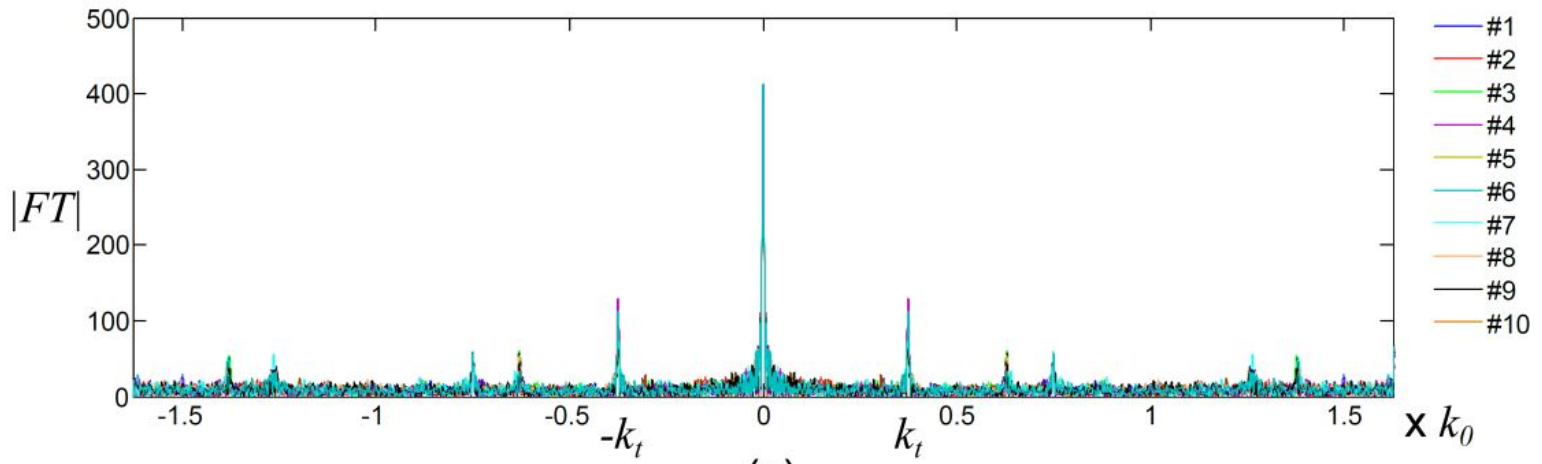

(c)

Figure S6. The ten different realizations for the design target of Figure 3(a) of the paper. (a) The displacement of each element with respect to the corresponding element in the reference design. (b) The absolute value of the Fourier transform of the lines demonstrates that different realizations are highly correlated in spectral domain. The spikes highlight the existence of required spatial frequencies including the targeted angular direction in the far-field, denoted by kt, and the guided waves components (multiples of $\mathrm{kg}=1.62 \mathrm{k}$ ). (c) The same plot as (b) from $-\mathrm{kg}$ to $+\mathrm{kg}$ (inside the dotted lines in (b)). 


\section{References:}

(1) Casati, G.; Molinari, L.; and Izrailev, F. Scaling properties of band random matrices. Phys. Rev. Lett. 1990, 64, 1851.

(2) Katagi, T.; Mano, S.; Sato, S.I. An improved design method of Rotman lens antennas. IEEE Trans. on Antennas and propag. 1984, 32, 524-527.

(3)Li, Y.B.; Cai, B.G.; Cheng, Q.; Cui, T.J. Isotropic Holographic Metasurfaces for Dual-Functional Radiations without Mutual Interferences. Adv. Funct. Mater. 2016, 26,29-35.

(4) Haghtalab, M.; Safavi-Naeini, S. Freeform engineered disordered metalenses for superresolution imaging and communication. Opt. Express 2018, 26, 9749-9771.

(5) Yang, J.; Fan, J.A. Topology-optimized metasurfaces: impact of initial geometric layout. Opt. Lett. 2017, 42, 3161-3164. 